San Jose State University

SJSU ScholarWorks

Faculty Publications, Chemistry

Chemistry

$1-1-1981$

\title{
Conduction Band Symmetry in Ta Chalcogenides from Ta L Edge X-ray Absorption Spectroscopy (XAS)
}

Juana Vivó Acrivos

San Jose State University, juana.acrivos@sjsu.edu

S.S. P. Parkin

University of Cambridge

J. Code

San Jose State University

J. Reynolds

San Jose State University

K. Hathaway

San Jose State University

See next page for additional authors

Follow this and additional works at: https://scholarworks.sjsu.edu/chem_pub

Part of the Physical Chemistry Commons

\section{Recommended Citation}

Juana Vivó Acrivos, S.S. P. Parkin, J. Code, J. Reynolds, K. Hathaway, H. Kurasaki, and E. Marseglia.

"Conduction Band Symmetry in Ta Chalcogenides from Ta L Edge X-ray Absorption Spectroscopy (XAS)" Journal of Physics C: Solid State Physics (1981): L349-L357. https://doi.org/10.1088/0022-3719/14/11/ 014

This Article is brought to you for free and open access by the Chemistry at SJSU ScholarWorks. It has been accepted for inclusion in Faculty Publications, Chemistry by an authorized administrator of SJSU ScholarWorks. For more information, please contact scholarworks@sjsu.edu. 
Authors

Juana Vivó Acrivos, S.S. P. Parkin, J. Code, J. Reynolds, K. Hathaway, H. Kurasaki, and E. Marseglia 
J. Phys. C: Solid State Phys, 14 (1981) L_349-L357. Printed in Great Britain

\title{
LETTER TO THE EDITOR
}

\section{Conduction band symmetry in Ta chalcogenides from Ta L edge x-ray absorption spectroscopy (XAs)}

\author{
J V Acrivos $\nmid$, S S P Parkinł, J Code $\nmid$, J Reynolds $\dagger$, K Hathawaył, \\ H Kurasaki $\dagger$ and $\mathrm{E} \Lambda$ Marsegliał \\ + San Jose State University, San Jose, CA 95912, USA \\ 1: Cavendish Laboratory, University of Cambridge, Madingley Road, Cambridgc CB3 \\ OHIE, UK
}

Received 4 February 1981

\begin{abstract}
We report measurements of the $\mathrm{x}$-ray absorption spectra near the $\mathrm{Ta} \mathrm{L}$ edges of different polytypes of $\mathrm{TaS}_{2}$ and $\mathrm{TaSe}_{2}$ and $\mathrm{TaSe}_{3}$. Analysis of structure seen near the absorption edges and associated with transitions from well defined core levels to conduction band states has cnabled the extent of the $p$ character of the predominantly $d$ character conduction band to be probed. The results show that the conduction band in the Ta chalcogenides studies has a significant amount of p character. and a lower limit of between 6 and $10 \%$ has been determined. The amount of pcharacter varies between the various compounds in the following way:
\end{abstract}

$$
1 \mathrm{~T}-\mathrm{TaS}_{2}<\mathrm{TaSe}_{3}<2 \mathrm{H}-\mathrm{TaS} 2-1 \mathrm{~T}-\mathrm{TaSe}_{2}<2 \mathrm{H}-\mathrm{TaSe}_{2} .
$$

The Ta $\mathrm{x}$-ray absorption edges have also been monitored during and alter intercalation of the chalcogenides with hydrazine. Small changes in p character are observed and the results indicate that intercalation modifies the conduction band. not only through charge transfer, but more significantly through changes in hybridisation of the band.

In the layered transition metal dichalcogenides (TMDC) there is a long standing question concerning the extent of hybridisation of the metal d states with the chalcogen $p$ states in the formation of the conduction band (schematic energy band structures for these compounds are given in Bell and Liang (1976); for a recent review of band structure calculations see, for example, Doran (1980) and references therein). Direct experimental data on the amount of hybridisation would be useful in the calculation of band structures of these materials, and in a fundamental understanding of those properties which depend on the bonding between the metal and chalcogen atoms. For example, the amount of hybridisation affects the interpretation of certain features in the measured reflectivity spectra of the group V(a) TMDC (Parkin and Beal 1980) and of excitonic features seen in the spectra of some of the group VI $(a)$ dichalcogenides (Goldberg et al 1975).

Identification of the symmetry allowed transitions can be complicated in the visible-ultraviolet region because of the convolution of the valence band density of states (DOS) with the conduction band Dos on which the transition strength depends (see, for example, Liang and Bcal 1976) and in the soft x-ray region because of overlap of transitions from different core levels. In particular, the Xas measurements of Sonntag 
and Brown (1974) on $2 \mathrm{H}-\mathrm{NbSe}_{2}$, in the soft x-ray region, are complicated by the overlap of the Se $\left(\mathrm{M}_{\mathrm{V}}\right.$ and $\left.\mathrm{M}_{\mathrm{IV}}\right)$ and $\mathrm{Nb}\left(\mathrm{N}_{\mathrm{I}}\right)$ edges at 56.7 and $58.1 \mathrm{eV}$ respectively, although Sonntag and Brown have identified the strong absorption near $53-59 \mathrm{eV}$ as resulting from Se $\mathrm{M}_{\mathrm{V}}, \mathrm{M}_{\mathrm{IV}}$ core level transitions to the conduction band states.

We have made measurements of the $x$-ray absorption spectra at the tantalum $L$ edges $\left(\mathrm{L}_{\mathrm{I}}\right.$ at $11.68 ; \mathrm{L}_{\mathrm{II}}$ at $11.11 ; \mathrm{L}_{\mathrm{III}}$ at $9.88 \mathrm{keV}$ ) of different polytypes of $\mathrm{TaS}_{2}$ and $\mathrm{TaSe}_{2}$ and $\mathrm{TaSe}_{3}$ in order to shed light on the symmetry of the conduction band states in these materials. (Note that the Ta L edges are well separated in energy from the Se K edge near $12.65 \mathrm{keV}$ ). XAs measures transitions from narrow core levels in the crystal to states in the conduction band and to the continuum. By observing the strengths of these transitions, information can be obtained directly about the symmetry of the states in the conduction band, because the absorption coefficient is related to the simple product of the conduction band density of states $N_{\mathrm{c}}(E)$ and the transition probability $|M(E)|^{2}$. If the conduction band consists of hybridised states of $d$ and $p$ symmetry then the selection rules for transitions will be modified accordingly and this will affect the strength of the transition. The symmetry allowed transitions between atomic levels on a single atom are as follows: (i) from the $L_{\text {III }}$ edge, $p_{3 / 2} \rightarrow d$ and s; (ii) from the $L_{11}$ edge, $p_{1 / 2} \rightarrow d$ and $\mathrm{s}$; and (iii) from the $\mathrm{L}_{\mathrm{l}}$ edge, $\mathrm{s} \rightarrow \mathrm{p}$.

The Ta L cdges were measured at the Stanford Synchrotron Radiation Laboratory (SSRL) using procedures described elsewhere (see for example Lindau and Winick 1976 and Winick and Brown 1978). The photon fluxes used in these experiments were produced by the SPEAR storage ring running at $2 \mathrm{GeV}$ and between 8 and $14 \mathrm{~mA}$. The SSRL source with the experimental configuration used for this study is shown in figure 1. The crystalline samples were contained in an evacuated cell made from s/s 316 with $0.03^{\prime \prime}$ thick beryllium windows. A mask with an aperture smaller than the size of the

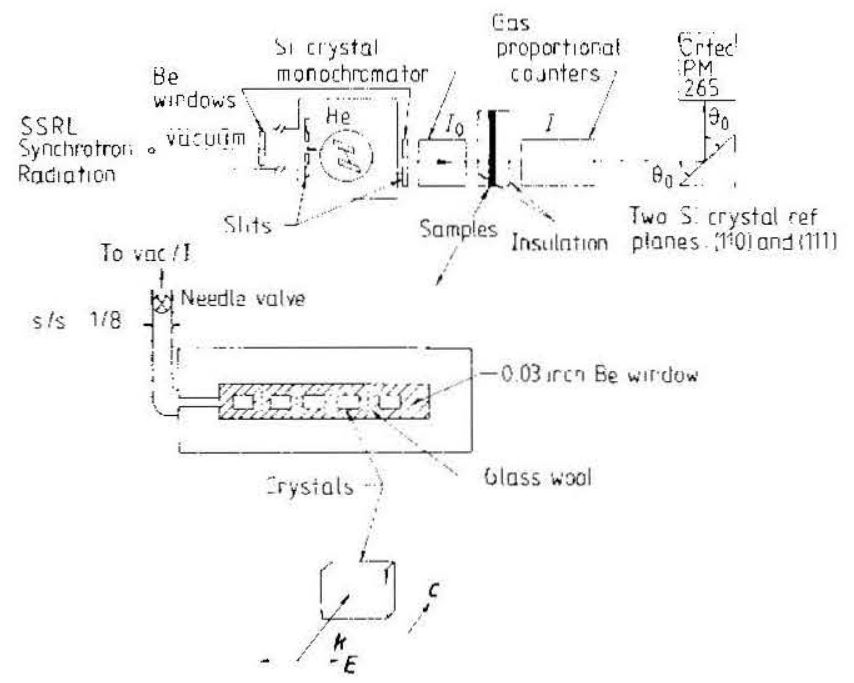

Figure 1. Schematic diagram of the experimental set-up. showing the SSRI. synchrotron radiation source, silicon crystal monochromator and gas proportional counters, placed cither side of the samples. Glass wool was used to hold the samples in place within an evacuated stainless steel cell containing beryllium windows, transparent in the energy range of interest. Bragg reficctions from two silicon crystals cut along the (111) and (110) planes were used to determine accurately an energy reference marker for cach sean, as deseribed by Acrivos et al (1980).

Table 1. taken $\mathrm{fr}$

\begin{tabular}{l} 
Edge \\
\hline $\mathrm{L}_{1}$ \\
$\mathrm{~L}_{\mathrm{II}}$ \\
$\mathrm{L}_{\mathrm{III}}$ \\
\hline
\end{tabular}

smallest crystal (app beam and each cryst. absorbance at a part cleaving the single cr ratio of incident to $t$ $A \equiv \ln \left(I_{0} / I\right) \sim 2-3, \mathrm{c}$ coefficients near the The $x$-ray bcam from propagation vector structure and the ext later. In these exper $\mathrm{TaS}_{2}$ or $\mathrm{TaSe}_{2}$ layers better than $0.7 \mathrm{eV}$, w in figures 2 and 3 (A resolution of the $\mathrm{L} \mathrm{ce}$ of a particular symm elements (Parratt 19 $\simeq 6 \mathrm{eV}$ for the Ta $L_{I I}$ lines have a similar $F$ of the experiments, $\mathrm{s}$ (of total width $\sim 7 \mathrm{eV}$ soft $x$-ray region the about $0.2 \mathrm{eV}$; but th: spectrum is more con

The applicability energies has been exa the refractive index $n$ $1-n \equiv \delta$ and both $\delta$ near the $\mathrm{x}$-ray absorp that $\bar{n}$ and $\bar{\varepsilon}$ are given

$$
\tilde{n} \equiv 1
$$

and

$$
\tilde{\varepsilon} \equiv n^{2}
$$

If interference effect incident light parallel

$$
T(E)=
$$


iplicated by the overlap respectively, although o $53-59 \mathrm{eV}$ as resulting ates.

at the tantalum L edges of $\mathrm{TaS}_{2}$ and $\mathrm{TaSe}_{2}$ and on band states in these gy from the $\mathrm{Se} K$ edge Is in the crystal to states the strengths of these letry of the states in the o the simple product of probability $\mid M(E)^{2}$. If netry then the selection fect the strength of the Icvels on a single atom e $\mathrm{L}_{\text {II }}$ edge, $\mathrm{p}_{1 / 2} \rightarrow \mathrm{d}$ and

Radiation Laboratory indau and Winick 1976 tese experiments were reen 8 and $14 \mathrm{~mA}$. The tudy is shown in figure made from $\mathrm{s} / \mathrm{s} 316$ with ler than the size of the

xing the SSRL synchrotron ional counters, placed either n place within an evacuated the energy range of interest. ad (110) plantes were used to as described by Acrivos et
Table 1. Tantalum mass absorption coefficients near the $\mathrm{L}$ edges in elemental tantalum. taken from the Chemical Rubber Company (CRC) handbook.

\begin{tabular}{lll}
\hline Edge & $\begin{array}{l}\alpha_{\mathrm{a}}^{1)} \\
\left(\mathrm{g} \mathrm{cm}^{2}\right)\end{array}$ & $\begin{array}{l}\Delta \alpha_{i}^{0} \\
\left(\mathrm{~g} \mathrm{~cm}^{-2}\right)\end{array}$ \\
\hline $\mathrm{L}_{\mathrm{l}}$ & 262 & 43 \\
$\mathrm{~L}_{\text {II }}$ & 248 & 70 \\
$\mathrm{~L}_{\text {III }}$ & 246 & 149 \\
\hline
\end{tabular}

smallest crystal (approximately $4 \mathrm{~mm}^{2}$ in cross section) was placed across the incident beam and each crystal was located with translational step motors so as to maximise the absorbance at a particular energy near $\mathrm{L}_{\mathrm{III}}$. The sample path length was established by cleaving the single crystal samples with the aid of adhesive tape, to achieve an optimum ratio of incident to transmitted intensities $I_{0} / I$ near the edge such that the absorbance $A \equiv \ln \left(I_{0} / I\right) \sim 2-3$, corresponding to thicknesses of $\simeq 25 \mu \mathrm{m}$. The Ta mass absorption coefficients near the L edges are given in table 1 , and are taken from the CRC handbook. The x-ray beam from a synchrotron is highly polarised in one direction $E$ normal to the propagation vector $\boldsymbol{k}$, which can be important in the interpretation of both the edge structure and the extended $x$-ray absorption fine structure (FXAFS) which we shall report later. In these experiments $k$ is parallel to the crystal $c$ axis and perpendicular to the $\mathrm{TaS}_{2}$ or TaSe $\mathrm{T}_{2}$ layers for the dichalcogenide compounds. The instrumental resolution is better than $0.7 \mathrm{eV}$, which is the width at half-height of the silicon diffraction references in figures 2 and 3 (Acrivos et al 1980). Initial and final state lifetimes will decrease the resolution of the Ledge measurements (Parratt 1959, Brown 1974). The width of a level of a particular symmetry increases monotonically with photon energy for the lighter elements (Parratt 1959) giving, by extrapolation, a full width at half height (FwHI) of $\simeq 6 \mathrm{eV}$ for the Ta $\mathrm{L}_{\text {III }}$ level. This is in agreement with our data, for which the sharpest lines have a similar FWHH. Thus the short lifetimes of the Ta $\mathrm{L}$ levels limit the resolution of the experiments, so much so that fine features in the conduction band density of states (of total width $\sim 7 \mathrm{eV}$ for the Ta dichalcogenides) cannot be observed. Note that in the soft $\mathrm{x}$-ray region the Fwin is very much smaller, and in particular for the $\mathrm{Se} \mathrm{M}_{\mathrm{V}}$ level is about $0.2 \mathrm{cV}$; but that, as mentioned previously, the interpretation of the absorption spectrum is more complicated at low energies because of overlap of absorption edges.

The applicability of the electromagnetic theory of absorption spectroscopy at $\mathrm{x}$-ray energies has been examined by Rehn (1979). In the x-ray spectral region the real part of the refractive index $n \approx 1$, and the extinction coefficient $\kappa \ll 1$. Rehn (1979) has defined $1-n \equiv \delta$ and both $\delta$ and $\kappa$ decrease monotonically with the photon energy $E$, except near the $x$-ray absorption edge where quantum conditions are satisfied. It thus follows that $\tilde{n}$ and $\tilde{\varepsilon}$ are given approximately by

$$
\tilde{n} \equiv 1-\delta+\mathrm{i} \kappa-1
$$

and

$$
\tilde{\varepsilon} \equiv n^{2}-\kappa^{2}+2 \mathrm{i} n \kappa \approx 1-2 \delta+2 \mathrm{i} \kappa .
$$

If interference effects may be neglected, the transmittance of our thin samples for incident light parallel to the $c$ axis is given by the usual relation,

$$
T(E)=\frac{I}{I_{0}}-\frac{\left(1+\kappa^{2} / n^{2}\right)\left(1-R_{0}\right)^{2} \exp (-\overline{\mu t})}{1+R_{0}^{2} \exp (-2 \overline{u t})}
$$


where the reflectance $R_{0}$ is given by

$$
R_{0}(E)=\frac{(n-1)^{2}+\kappa^{2}}{(n+1)^{2}+\kappa^{2}} \sim \frac{\delta^{2}+\kappa^{2}}{4}
$$

in the above approximation and where $\mu=4 \pi \kappa / \lambda$, and $\overline{\mu t}$ is defined by

$$
\overline{\mu t}=\alpha_{\mathrm{a}}(E) \rho_{\mathrm{a}} t_{\mathrm{a}}+\sum_{i \neq a} \alpha_{i}(E) \rho_{i} t_{i}=A_{\mathrm{a}}+\Lambda_{\mathrm{bg}}
$$

where the $\alpha(E)$ are experimental mass absorption coefficients (given, for example, in the Chemical Rubber Company handbook) for the matter in the x-ray path of length $t$, and $\rho$ is the associated density. We distinguish the Ta atoms by the subscript a. Below the edge we define $\alpha_{\mathrm{a}}=\alpha_{\mathrm{a}}$ and above the edge $\alpha_{\mathrm{a}} \equiv \alpha_{\mathrm{a}}$, so that at the absorption edge $E_{\mathrm{a}}$ there is a discontinuity in $\overline{\mu t}$, the so-called edge jump, given by

$$
\Delta A=\left(\overline{\mu t}_{>}-\overline{\mu t}\right)_{E_{\mathrm{a}}}=\left(\alpha_{\mathrm{a}>}^{i !}-\alpha_{\mathrm{a}<}^{i !}\right) \rho_{\mathrm{ul}} t_{\mathrm{a}}
$$

where $\Delta \alpha_{\mathrm{a}}^{0}=\alpha_{\mathrm{a}>}^{0}-\alpha_{\mathrm{a}<}^{0}$ are given in table 1 for the three $\mathrm{L}$ edges for the elemental Ta standard. It follows from equations (3) and (4) that within the above approximation

$$
\overline{\mu t}=\ln \left(I_{1} / I\right) .
$$

Figure 2 shows plots of transmittance against energy for a typical compound near the $\mathrm{L}_{\mathrm{I}}, \mathrm{L}_{\mathrm{II}}$ and $\mathrm{L}_{\mathrm{III}}$ edges. Similar results are obtained for all the unintercalated compounds shown in table 2. From equation (5) it follows that

$$
A=-\ln (T)=A_{\mathrm{a}}(E)+A_{\text {bg }}(E)
$$

where the background contribution $A_{\mathrm{bg}}$ can be subtracted out to obtain $A_{\mathrm{at}}(E)$ as follows:

$$
A_{\mathrm{at}}(E)=A_{\mathrm{at}}^{*}(E)\left[1-\chi_{\mathrm{as}}(E)\right]
$$

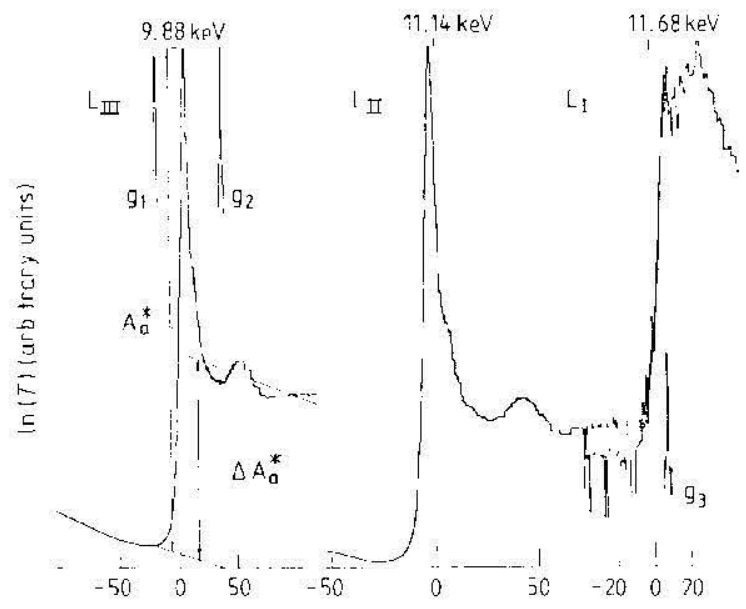

Figure 2. X-ray absorption spectra at the $\mathrm{Ta}$ L edges in $2 \mathrm{H}-\mathrm{TaS}_{2}$, showing the peaks associated with transitions into conduction band states, seen superimposed on the edge jump. $\Delta \boldsymbol{A}_{\text {ai }}$, the edge jump, is defined in the figure and $A_{\mathrm{a} i}^{*}$ is the magnitude of the superimposed peak. The results clearly show that the peak at the $\mathrm{L}_{j}$ edge is considerably smaller than that at the $\mathrm{L}_{\mathrm{II}}$ or $\mathrm{L}_{\mathrm{II}}$ edge, and indeed can only just be resolved. $\mathrm{g}_{i}$ are the $S$ i diffraction peak energy reference markers. Note that the energy is given with respect to the absorption edge position in pure tantalum.

Table 2. I measurem are the lea and its sizo

Compoun

$1 \mathrm{~T}-\mathrm{TaS}_{2}$

$2 \mathrm{H}-\mathrm{TaS}_{2}$

1T-TaSe,

$2 \mathrm{H}-\mathrm{IaSc}$

$\mathrm{TaSe}_{3}$

$3 \mathrm{R}-\mathrm{TaS}_{2} \cdot$

$2 \mathrm{H}_{\mathrm{i}}-\mathrm{TaS}_{2}$

$x \mathrm{TaSe}_{2} \cdot \mathrm{I}_{r}$

$x \mathrm{TaSe}_{2} \cdot \mathrm{I}_{2}$ (2H)

$\mathrm{TaSc}_{3} \cdot \mathrm{I}_{1: 3}$

$2 \mathrm{H}-\mathrm{TaS}_{2} \cdot$

2H-TaS

$2 \mathrm{H}-\mathrm{TaS}_{2} \cdot \mathrm{I}$

where $A_{\mathrm{a}}^{*}(E)$ correspor and continuum states a

Additional peaks nc 2 are identified with tra measured with respec $A_{\mathrm{al}}^{*} / \Delta A_{a 1}^{*}$ are compare ratios for either the $\mathrm{L}_{\mathrm{II}}$ $1 \mathrm{~T}-\mathrm{TaS}_{2}$ 
Table 2. Intensities of the additional peaks secn near the absorption edges in the XAS measurements, measured with respee to the edge jump. Note that the $l_{-}$edge measurements are the least reliable because the peak is of the same order of magnitude as the edge jump and its size will depend sensitively on its position with respect to the edge.

ined by

\begin{tabular}{|c|c|c|c|}
\hline Compound & L. edge & Run number & $A_{i 1}^{2} / \Delta A_{: 11}$ \\
\hline \multirow[t]{3}{*}{$1 \mathrm{~T}-\mathrm{TaS}_{2}$} & 1 & $V 208.9$ & $\geqslant 0.5$ \\
\hline & II & V2014 & 3.3 \\
\hline & III & $V 211$ & 2.9 \\
\hline \multirow[t]{3}{*}{$2 \mathrm{H} \cdot \mathrm{TaS}_{2}$} & 1 & $V 225.6$ & $\geq 0.5$ \\
\hline & II & V121, 1 & 3.1 \\
\hline & III & V 1.52 .3 & 2.5 \\
\hline \multirow[t]{3}{*}{ 1T-TaSe } & I & $V 197$ & 20.5 \\
\hline & II & $V 192,3$ & 3.1 \\
\hline & III & $V 168,9$ & 2.2 \\
\hline \multirow[t]{3}{*}{$2 \mathrm{H}-\mathrm{TaSe}_{2}$} & I & $\vee 228,9$ & $\geqslant 0.5$ \\
\hline & II & $V 234,5$ & 2.6 \\
\hline & III & $\mathrm{V} 180,2$ & 1.8 \\
\hline \multirow[t]{3}{*}{$\mathrm{TaSc}_{3}$} & I & $V 246,7$ & $\geqslant 0.5$ \\
\hline & II & $V 139,140$ & 3.2 \\
\hline & III & $\mathrm{V} 142$ & 2.7 \\
\hline \multirow{3}{*}{$3 \mathrm{R}-\mathrm{TaS}_{2} \cdot \mathbf{l}_{4 ; 3}$} & 1 & $V 1205$ & $\geqslant 0.6$ \\
\hline & II & VI248 & 3.2 \\
\hline & 111 & VI204 & 3.2 \\
\hline \multirow[t]{3}{*}{$2 \mathrm{H}_{\mathrm{l}}-\mathrm{TaS}_{2} \cdot \mathrm{I}_{23}$} & I & VI 236.7 & $\geqslant 0.5$ \\
\hline & II & $V 1216,142$ & 3.1 \\
\hline & III & VI2018 & 2.9 \\
\hline$x \operatorname{TaSc}_{2} \cdot \mathbf{l}_{x}$ & 1 & $V 1238$ & $\geqslant 0.5$ \\
\hline \multirow{2}{*}{ (1T) } & II & V1239 & 3.0 \\
\hline & III & VI177 & 1.9 \\
\hline & 1 & VI240 & $\geqslant 0.5$ \\
\hline \multirow{2}{*}{$(2 \mathrm{H})$} & II & VI 242,3 & 3.2 \\
\hline & III & V1173.5 & 1.6 \\
\hline \multirow[t]{3}{*}{$\mathrm{TaSe}_{3} \cdot \mathrm{I}_{33}$} & 1 & VI 245,6 & $\approx 0.3$ \\
\hline & II & VI 23,4 & 3.5 \\
\hline & III & VI 247 & 2.8 \\
\hline \multirow{3}{*}{$2 \mathrm{H}-\mathrm{TaS}_{2} \cdot \mathrm{Cr}_{1 / 3}$} & I & VIM130 & $\geqslant 0.4$ \\
\hline & II & - & - \\
\hline & III & VIM123 & 2.2 \\
\hline \multirow[t]{3}{*}{$2 \mathrm{H}-\mathrm{TaS}_{2} \cdot \mathrm{Co}_{1 \cdot 3}$} & I & VIM125 & $\geqslant 0.5$ \\
\hline & II & VIM 127 & 2.1 \\
\hline & III & VIM 124 & 1.5 \\
\hline \multirow[t]{3}{*}{$2 \mathrm{H}-\mathrm{TaS}_{2} \cdot \mathrm{Ni}_{1 ; 3}$} & I & VIM116 & $\approx 0.5$ \\
\hline & II & - & - \\
\hline & [I] & VIM117 & 2.4 \\
\hline
\end{tabular}

(given, for example, in he x-ray path of length $t$, $y$ the subscript a. Below at at the absorption cdge by

ges for the elemental Ta above approximation

pical compound near the intercalated compounds obtain $A_{\mathrm{a}}(E)$ as follows:

, showing the peaks associated sed on the edge jump, $\Delta A_{i 1}$. de of the superimposed peak. erably smaller than that at the he Si diffraction peak cnergy o the absurption edge position 


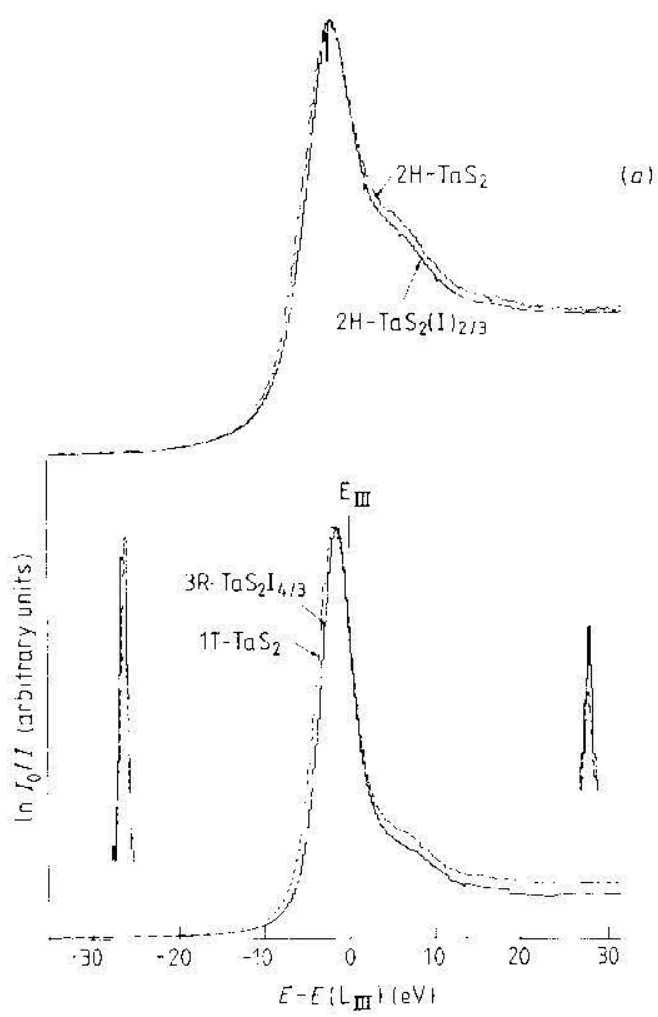

Intercatation of $1 T-T_{a} S_{2}$ an $x$-ray becm

Figure 3. $\mathrm{Ch}$ resulting fror $2 \mathrm{H}-\mathrm{TaS}_{2}$ befe only small ch energies nea laken during clearly indica lhe intercala layers per $\mathrm{Ta}$ the two interc on pumping, in the interca host compour infrared tran munication). $2 \mathrm{H}-\mathrm{TaSe}_{2}$ anc Ta selenides the Ta $L_{I}$ edgo by intercalatic as at shoulder 

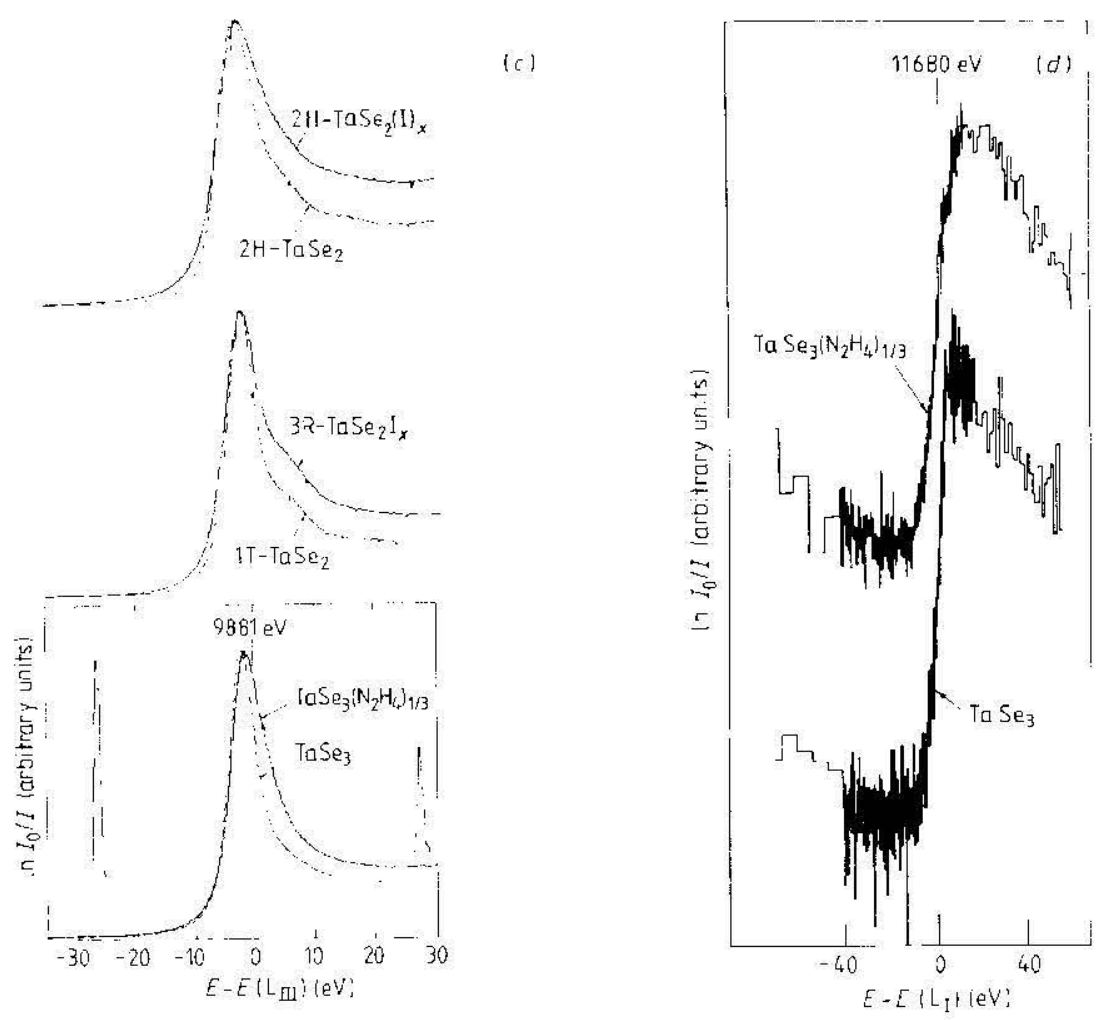

Figure 3. Changes in the Ta $\mathrm{L}$ edge $\mathrm{x}$-ray absorption spectra in several Ta chalogenides. resulting from hydrazine $\left(\mathrm{N}_{2} \mathrm{H}_{1}\right)$ intercalation. (a) $\mathrm{XAS}_{\mathrm{A}}$ at the Ta $\mathbf{L}_{\mathrm{III}}$ edge in $1 \mathrm{~T}-\mathrm{TaS} \mathrm{S}_{2}$ and $2 \mathrm{H}-\mathrm{TaS}_{2}$ before and after intercalation with hydrazine. Note that the intercalation causes only small changes in the spectra of these sulphides. (b) A detail of the $I_{\text {.11 }}$ edge Xas for energies near the peak position for intercalation of $1 \mathrm{~T}$-TaS $\mathrm{T}_{2}$. More than 40 spectra were taken during and prior to intercalation of $1 \mathrm{~T}-\mathrm{TaS}_{2}$ with $\mathrm{N}_{2} \mathrm{H}_{4}$ to form $3 \mathrm{R}-\mathrm{TaS}_{2}\left(\mathrm{~N}_{2} \mathrm{H}_{4}\right)_{4,3}$, clearly indicating that the peak position is shilted to higher energies in $3 \mathrm{R}-\mathrm{TaS}$ ? $\left(\mathrm{N}_{2} \mathrm{H}_{4}\right)_{433}$. The intercalation complex formed in this way, $3 \mathrm{R}-\mathrm{TaS}_{2}\left(\mathrm{~N}_{2} \mathrm{H}_{1}\right)_{4: \text {, contains two intercalate }}$ layers per $\mathrm{TaS}_{2}$ layer (Acrivos 1979). Pumping on the intercalated sample removes one of the two intercalate layers to give $3 \mathrm{R}-\mathrm{TaS}_{2}\left(\mathrm{~N}_{2} \mathrm{H}_{4}\right)_{23}$ (Acrivos 1979). The xAs spectra obtained on pumping, shown in the figure, indicates that the peak position is shifted to lower energies in the intercalation complex, $3 \mathrm{R}-\mathrm{TaS}_{2}\left(\mathrm{~N}_{2} \mathrm{H}_{4}\right)_{-3}$, even as compared with the unintercalated host compound, 1T-TaS,. The signs of the shifts are consistent with the changes seen in the infrared transmission spectra, induced by hydrazine intercalation (M Sarma private communication). (c) XAs spectra at the Ta $\mathrm{L}_{\text {III }}$ edge belore and after intercalation of $1 \mathrm{~T}^{-}-\mathrm{TaSe}_{2}$, $2 \mathrm{H}-\mathrm{TaSe}_{2}$ and $\mathrm{TaSe}_{3}$ with $\mathrm{N}_{2} \mathrm{H}_{4}$. Note that much larger changes are seen in the spectra of the Ta selenides as compared to the Ta sulphides (see figures $3(a)$ and $3(b)$ ). (d) XAs spectra at the $\mathrm{Ta}_{2} \mathrm{~L}_{1}$ edge in $\mathrm{TaSc}_{3}$, showing a significant change in the magnitude of the $\mathrm{L}_{1}$ peak induced by intercalation. The peak can be scen above the edge jump in the pure compound. but only as a shoulder in the intercalated material. 
in rough agreement with the order expected from chemical arguments. On intercalation with $\mathrm{N}_{2} \mathrm{H}_{4}$ the ratio decreases for the three selenides but it increases for the disulphides. However, for the $\mathrm{Cr}$, $\mathrm{Co}$ and $\mathrm{Ni}$ intercalates of $2 \mathrm{H}-\mathrm{TaS}$, the ratio is decreased in all cases with the greatest change observed for the Co intercalate. These results will be presented in more detail in a separate publication.

The oscillator strengths can be compared by taking into account the dependence of $\Delta A_{\mathrm{a}}^{*}$ on $\Delta \alpha_{\mathrm{a}}^{0}$ through equation (6). Given

$$
\left.\Delta \alpha_{\mathrm{a}}^{0} \rho_{\mathrm{i}} t_{\mathrm{a}} \propto M_{\mathrm{a}}^{0}\right|^{2} / E_{\mathrm{a}}^{2}
$$

where $M_{\mathrm{a}}^{0}$ is the matrix element for the transition probability from the $\mathbf{L}$ core states to the continuum (in pure tantalum), excluding transitions to bound states, it follows that

$$
N_{\mathrm{c}}\left|M_{\mathrm{a}}\right|^{2} \propto \Delta \alpha_{\mathrm{a}}^{0} E_{\mathrm{a}}^{2}\left(A_{\mathrm{a} 1}^{*} / A_{\mathrm{a}}^{*}\right)
$$

where $N_{\mathrm{c}}$ is the density of final states and $M_{\mathrm{i}}$ the matrix element for the transition probability from the corresponding L core state to conduction band states. Using relation (10) we have estimated the relative amount of $\mathrm{p}-\mathrm{d}$ character in the final states using the relation

$$
r_{i}=\frac{\left(N_{\mathrm{c}}\left|M_{\mathrm{a}}\right|^{2}\right)_{\mathrm{pstates}}}{\left(N_{\mathrm{c}}\left|M_{\mathrm{a}}\right|^{2}\right)_{\mathrm{d} \text { tates }}}=\frac{\Delta \alpha_{\mathrm{al}}^{\mathrm{j}} E_{\mathrm{l}}^{2}\left(A_{\mathrm{a} 1}^{*} / \Delta A_{\mathrm{i}}^{*}\right)_{\mathrm{I}}}{\Delta \alpha_{\mathrm{a} i}^{01} E_{i}^{2}\left(A_{\mathrm{a} 1}^{*} / \Delta A_{\mathrm{a}}^{*}\right)_{i}}
$$

where $i=$ II or III. We now define the percentage p character of the final states as seen in this experiment by the following:

$$
p_{i}=100 r_{i} /\left(1+r_{i}\right) \text {. }
$$

Using the values $\Delta \alpha_{\mathrm{a} i}^{0} E_{i}^{2}=5.87 \times 10^{3}, 8.69 \times 10^{3}$ and $14.54 \times 10^{3} \mathrm{~cm}^{2} \mathrm{~g}{ }^{1}(\mathrm{keV})^{2}$ for the $\mathrm{L}_{\mathrm{I}}, \mathrm{L}_{\mathrm{II}}$ and $\mathrm{L}_{\mathrm{III}}$ edges respectively and values for $A_{\mathrm{n}}^{*} / \Delta A_{\mathrm{a} 1}^{*}$ given in table 2 via relation (11), we find that $p_{\mathrm{II}}$ and $p_{\mathrm{II}}$ are similar and lie in the range $6-10 \%$.

In order to compare these results with band structurc calculations the nature of the transition moments must be examined. The matrix element $M_{\mathrm{a}}$ for the transition can be written as a product of a term which depends only on the symmetry of the initial and final states and a term which depends on the spatial distributions of these states (Wigner-Eckart theorem). The symmetry-dependent term will, in principle, depend on the direction of the radiation field $E$ (see for example Tinkham 1964) and therefore the p-d character given in table 2 refers only to the symmetry-allowed transitions under the particular conditions of the experiment (namely $\boldsymbol{E}$ parallel to the $\mathrm{TaS}_{2}$ or $\mathrm{TaSc}_{2}$ layers for the dichalcogenides $(k \| c)$ ). When the final state is a hybrid of contributions from different states and the initial state is centred at only one atom, in this case $\mathrm{Ta}$, then the contributions to $M_{\mathrm{a}}$ will be weighted by the overlap of the initial state with the hybrid components of the final state. Thus the p character given by relation (12) is a measure of the Ta $\mathrm{p}$ character in the conduction band plus a small contribution due to overlap with chalcogen states, not necessarily of p character. Therefore it follows that the total p character of the band is larger than that reported above.

Although in practice insufficient flux is available at the SSRI, in principal similar measurements at the chalcogen L edges would reveal the amount of chalcogen $p$ character in the conduction band together with some contribution duc to overlap of the initial state with states centred on the Ta atoms. (In $2 \mathrm{H} \mathrm{NbSe} \mathrm{N}_{2}$ we estimate the contribution due to overlap with the transition metal atom to be about $10 \%$ from the overlap of the $\mathrm{Nb} 4 \mathrm{~d}$ and Se portates of 0.31 calculated by Doran et al 1978.) Thus it would be possible to determine band from measurcm

The conduction $b$ character, although it between 6 and $10 \%$. in the conduction ban optical spectra. For ex unoccupied higher-en wise be the case if the interpretation of the $r$ transition metal inter

There are interesti conduction band is rec 3). Moreover, in this ce Such a shift could be $t$ by charge transfer fro transition to lower ene which shifts the transit the conduction band hybridisation of the ba

We thank A R Beal fo for helpful discussions at SJSU and NSF DN Energy, and a NATO edges a travel grant fro

\section{References}

Acrivos J V 1979 Physics an F Levy (Dordrecht: Re Acrivos J Vet al 1980 Stanfo Bell M G and I iang W Y 19 Brown F C 1974 Solid State vol 29

Doran N J 1980 Proc. Int. C $\mathrm{C}$ Haas and $\mathrm{H}$ W Myro Doran N J, Ricco B, Titterir Goldberg A M, Beal A R, L Liang $W Y$ and Bcal A R 19 Lindau I and Winick $\mathrm{H} 197 \mathrm{f}$ 1976 p 215

Parkin S S P and Beal A R 1 Parratt L G 1959 Rev. Mod. Rehn 1979 SSRL Report

Sonntag B and Brown F C 1 Tinkham M 1964 Group The Winick $\mathrm{H}$ and Brown $\mathrm{G}$ (ed) 
uments. On intercalation eases for the disulphides. e ratio is decreased in all ate. These results will be

count the dependence of

from the $\mathrm{L}$ core states to und states, it follows that

lement for the transition and states. Using relation the final states using the

of the final states as seen

$\times 10^{3} \mathrm{~cm}^{2} \mathrm{~g}^{-1}(\mathrm{keV})^{2}$ for iven in table 2 via relation $10 \%$.

lations the nature of the for the transition can bc nmetry of the initial and ibutions of these states , in principle, depend on 1 1964) and therefore the ved transitions under the the $\mathrm{TaS}_{2}$ or $\mathrm{TaSe}_{2}$ layers id of contributions from in this case $\mathrm{Ta}$, then the tial state with the hybrid elation (12) is a measure tribution due to overlap e it follows that the total

$\mathrm{RL}$, in principal similar unt of chalcogen p char$n$ due to overlap of the 2 we estimate the contriut $10 \%$ from the overlap 1978.) Thus it would be possible to determine a maximum value for the amount of $\mathrm{p}$ character in the conduction band from measurements at the chalcogen and metal Ledges.

The conduction band in the Ta chalcogenides studied has a significant amount of $p$ character, although it has only been possible to deduce a lower limit on this amount of between 6 and $10 \%$. These are the first direct measurements of the extent of p character in the conduction band of these compounds and may assist in the interpretation of their optical spectra. For example, transitions between occupied conduction band states and unoccupied higher-energy band states of $d$ character are not forbidden as would otherwise be the case if the conduction band were purely of $d$ symmetry, which supports the interpretation of the reflectivity spectra of the group $\mathrm{V}(a)$ dichalcogenides and their $3 \mathrm{~d}$ transition metal intercalates given by Parkin and Beal (1980).

There are interesting effects on intercalation. For example, the Ta p character of the conduction band is reduced in going from $\mathrm{TaSe}_{3}$ to $\mathrm{TaSe}_{3}\left(\mathrm{~N}_{2} \mathrm{H}_{4}\right)_{1,3}$ (see table 2 and figure 3). Moreover, in this compound the $\mathrm{L}_{\mathrm{III}}$ edge is shifted to higher energies on intercalation. Such a shift could be the result of two competing effects: (i) shielding of the core levels by charge transfer from the intercalate to the conduction band which would shift the transition to lower energy; and (ii) filling of the conduction band through charge transfer which shifts the transition to higher energies. Thus it appears that intercalation modifies the conduction band not only through charge transfer but also through changes in hybridisation of the band.

We thank A R Beal for providing crystals and are grateful to A D Yoffe and W Y Liang for helpful discussions. This work was partly supported by contracts NSF DMR7910011 at SJSU and NSF DMR7727489 at SSRL in cooperation with the US Department of Energy, and a NATO grant 1441 at the Cavendish Laboratory. S S P Parkin acknowledges a travel grant from Trinity College, Cambridge.

\section{References}

Acrivos I V 1979 Physics and Chemistry of Materials with Lavered Sirtctures: Intercalated Layer Materials ed F Levy (Dordrecht: Reidel) p 94

Acrivos J V et al 1980 Stanford Synchrotron Radiation I aboratory Repon

Bell M G and Liang W Y 1976 Adv. Phys. 2553

Brown F C 1974 Solid State Physics ed H Ehrenreich. F Seitz and D Turnbull (New York: Academic Press) $\operatorname{vol} 29$

Doran N J 1980 Proc. Int. Conf. on Layered Materials and Intercalates, Nijmegen 1979 ed C F van Bruggen, C Haas and H W Myron (Amsterdam: North-Holland)

Doran N J. Ricco B. Titterington D J and Wexler G 1978 J. Phys. C: Solid State Phys. 11685

Goldberg A M, Beal A R, Levy F A and Davis E A 1975 Phil. Mag. 32367

Liang W Y and Beal A R 1976 J. Phys. C: Solid State Phys. 92823

Lindau I and Winick H 1976 Scienific and Industrial Applications of Small Accelerators, Fourth Conference $1976 \mathrm{p} 215$

Parkin S S P and Beal A R 1980 Phil. Mag. 42627

Parratt L G 1959 Rev. Mod. Phys. 31616

Reho 1979 SSRL Repori

Sonntag B and Brown F C 1974 Phys. Rev. B10 2300

Tinkham M 1964 (iroup Theory and Quantum Mechanics (Ncw York: McGraw-Hill)

Winick II and Brown G (ed) 1978 Stanford Synchrotron Radiation Laboratory Report no 7804 